\title{
Special Issue: Smart Flow Control in Micro Scale
}

\author{
Jin-yuan Qian ${ }^{1,2,3} \mathbb{D}$, Junhui Zhang ${ }^{2} \mathbb{D}$, Zan $\mathrm{Wu}^{3}$ and Bengt Sunden ${ }^{3, * \mathbb{C}}$ \\ 1 Institute of Process Equipment, College of Energy Engineering, Zhejiang University, Hangzhou 310027, \\ China; qianjy@zju.edu.cn \\ 2 State Key Laboratory of Fluid Power and Mechatronic Systems, Zhejiang University, Hangzhou 310027, \\ China; benzjh@zju.edu.cn \\ 3 Department of Energy Sciences, Lund University, P.O. Box 118, SE-22100 Lund, Sweden; \\ Zan.Wu@energy.lth.se \\ * Correspondence: bengt.sunden@energy.lth.se; Tel.: +46-462228605
}

Received: 28 April 2020; Accepted: 28 April 2020; Published: 8 May 2020

\section{Introduction}

Smart control processes have been proposed for many years, while for smart flow control-especially when "smart flow control" comes at the microscale-it turns out that many new innovations and enabling technologies are possible. For instance, precise flow rate in a microreactor means high reaction efficiency. Similarly, for micromixers, smart fluid control can improve the precise distribution of every constituent. Such systems are made up of micropumps, microchannels, and microvalves, etc.

In this Special Issue on "Smart Flow Control Processes at the Microscale", 33 papers have been published, ranging from studies of flow characteristics in pumps or valves, dynamic performances in roiling mill systems or jet systems, to optimal design of special components in smart control systems. The Special Issue is available online at the following link:

https://www.mdpi.com/journal/processes/special_issues/Flow_Micro_Scale.

The contributions are summarized in four parts as follows:

\section{Smart Flow Control in Pumps}

A pump is one of the most important devices in fluid transportation systems. Research aiming at the flow field and pressure characteristics is of great importance for improvement of the operating performance.

Bai et al. [1] numerically studied the influence of pressure fluctuations and unsteady flow patterns in a pump flow channel with different diffuser vane numbers. The results indicate that the lower number of diffuser vanes was beneficial to obtain weaker pressure fluctuation intensity.

Cao et al. [2] investigated the whole flow field of a low specific speed centrifugal pump with five blades at different flow rates in order to study the near-wall region flow characteristics in a low-specific-speed centrifugal impeller. The main contribution of this work is the illustrations of pressure distribution and relative velocity distribution profiles on the pressure side of different blades.

Si et al. [3] studied the mechanism of radiated noise and its relationship with hydraulics in centrifugal pumps via a numerical method combined with an experimental approach. The results reveal that the radiated noise exhibits a typical dipole characteristic behavior and its directivity varies with the flow rate. In addition, Si et al. [4] carried out an experimental and numerical study aiming at the internal flow characteristics under gas-liquid two-phase flow in a miniature drainage pump. The pump performance and emitted noise measurements were monitored at various conditions. The study is a good reference for low noise design of drainage pumps.

Wang et al. [5] also analyzed the gas-water two-phase flow in a self-priming centrifugal pump. The results illustrate the three stages in a self-priming process. The effect of the middle stage is highlighted, which further determines the length of the self-priming time. 
Jiao et al. [6] studied the three-dimensional cavitation flow in a waterjet propulsion pump based on Zwart-Gerber-Belamri cavitation model and the RNG (Renormalization Group) k- $\varepsilon$ model. The study demonstrates that the potential dangerous regions of cavitation are the lip of the inlet passage and the upper and lower connecting curved section of the inlet passage.

Luo et al. [7] analyzed the unsteady flow process in waterjet pumps to improve the overall performance and optimization of the structure design. The surface vortex of the blade and the unsteady flow process of the propulsion pump at different times of the same period were demonstrated.

Cao et al. [8] studied the evolution of vortex structures in a laminar boundary layer over a flat plate by the Fourier spectral hybrid method. Results show that the maximum amplitudes of the vortex structures experience a process of linear growth and nonlinear rapid growth. The change in the mean flow profile further induces or promotes the growth or formation of vortex structures.

Jin et al. [9] investigated the external flow characteristics and pressure fluctuation in a submersible tubular pumping system. Results indicate that the pressure pulsation is less affected by the blade frequency with an increase of the measuring point from the impeller.

Xue et al. [10] proposed a design method based on Amesim and a Python script for the purpose of multi-objective optimization in static and dynamic performances of a pump-driven actuator. The mapping between the design parameters and the relations between the objectives are plotted. The results highlight the feasibility of the proposed method in achieving the multi-objective optimization.

Zhang et al. [11] investigated the structural characteristics of an ultra-high pressure axial piston pump. Via an analysis of the oil film pressure and thickness in different rotating angles of the piston-cylinder pair, it was found that the oil film pressure achieves the maximum value when the rotating angle increases to $90^{\circ}$, while the film thickness reaches the minimum at the same time.

Zheng et al. [12] provided a fluid pressure signal method for hydraulic pumps based on Autogram for solving the fluid pressure fluctuations caused by the center spring wear faults. The results highlight the superiority of standard Autogram on the extraction of fault feature information on center spring wear when comparing with upper Autogram and lower Autogram. Moreover, a novel method named as improved wavelet transform (IEWT) was proposed by Zheng et al. [13] in order to solve the segment over-decomposition obtained by the empirical wavelet transform (EWT). The proposed method was shown to be superior for eliminating the over-decomposition of the fault feature information.

\section{Smart Flow Control in Valves}

Valves play a significant role to change the flow rate, pressure and directions of fluids. Smart valves can turn out smart control of fluids.

Lei et al. [14] suggested a novel method depending on the Machine Learning Service (MLS) HUAWEI CLOUD to achieve accurate diagnosis of hydraulic valve faults. The method combines advantages of Principal Component Analysis (PCA) in dimensionality reduction and the eXtreme Gradient Boosting (XGBoost) algorithm and proves to be highly effective for identifying valve faults in the hydraulic directional valve.

Liu et al. [15] studied the throttling characteristics of the diaphragm valve. In order to identify the optimal design of the flow path profile, two-dimensional simulation of the Weir diaphragm valve flow field was conducted. The study shows that the flatting of the ridge side wall, widening of the ridge top and the gentle flatting of the internal protruding of the flow path prove to be three positive approaches for the improvement of the throttling characteristics.

Lu et al. [16] presented an investigation aiming at the oscillating flow field of the double-nozzle flapper servo valve pre-stage through Large Eddy Simulation (LES) turbulent modeling. Meanwhile, the User-Defined Function (UDF) was introduced to control the main stage movement. The results highlight the structure and flow parameter effect on the oscillating flow. In order to illustrate the damage caused by the increase of the injection pressure in the high pressure pump unloading valve ball, a theoretical calculation of the pressure relieve valve and the fatigue numerical simulation was carried out by Lu et al. [17]. Results indicate that the high pressure relief valve ball in the direct injection high 
pressure pump should not be a traditional structural damage under high pressure conditions and the surface damage of the valve ball is microscopic damage, such as fretting wear.

Qiu et al. [18] investigated the pressure drop and cavitation characteristics in the sleeve-regulating valve in different pressure differences and valve core displacements using the multiphase cavitation model. The results show that the decrease of the valve core displacement induces the enlargement of the vapor distribution region and the increase of the vapor density. The effects of the pressure difference on the cavitation intensity are more prominent with the decrease of the valve core displacement. The work provides valuable instructions for the cavitation control of the sleeve regulating valves.

Wu et al. [19] studied the flow and loss coefficients in a wedge-type double disk parallel gate valve. Effects of the Reynolds number, valve opening degree and groove depth were analyzed. The results suggest that a large groove depth should be selected to provide a large flow coefficient during the design process. However, during the machining process, the machining accuracy should be satisfied in order to avoid stress concentration of the bolt.

Besides, bileaflet mechanical heart valves (BMHVs) are widely used as the alternatives of diseased heart valves. Xu et al. [20] performed simulations of unsteady flow in a BMHV and pressure pulsation characteristics under different flow rates and leaflet fully opening angle conditions were investigated. The work provides a good reference for the alleviation of leaflet vibration phenomenon in BMHVs.

\section{Smart Flow Control in Microfluidics}

Droplet flow and microflow control in microfluidics are extensively studied.

Qian et al. [21] investigated the characteristics of droplets in a dynamic injection flow rate by the Volume of Fluid (VOF) method combined with UDF. The study presents a novel aspect of the droplet flow since the droplet generation is always at a constant flow rate of two phases in most researches.

Zhang et al. [22] studied the hydrodynamics of droplets passing through metal foam by the lattice Boltzmann method (LBM). The critical capillary number was identified. Results show that the droplet continues to be deformed until it breaks up when the capillary number is larger than 0.61 . In order to avoid the calescence of the adjacent droplet, the distance between the droplets should be larger than three times the diameter of the droplet.

$\mathrm{Li}$ et al. [23] investigated the two-phase flow inside a grooved rotating-disk system both in experimental and numerical methods. Visualization tests indicated that the flow field of the system was an air-oil flow. The stable interface between the continuous oil phase and the two-phase area could be formed and observed.

Guan et al. [24] proposed a miniaturized, easily processed, and inexpensive xenon micro flow control device (XMFCD) in order to reduce the volume and weight of the traditional XMFCDs. The design of the proposed XMFCD is based on complex three-dimensional (3D) microfluidic channels while the fabrication process is based on low-temperature co-fired ceramic (LTCC) technology and it was illustrated in detail.

\section{Smart Flow Control in Mechatronic Systems}

In mechatronic systems, there are many flow control issues, and smart flow control can improve the efficiency of mechatronic systems significantly.

A rolling mill with a hydraulic system is widely used in the strip steel industry. The vertical vibration seriously affects the stability of the rolling mill system. Zhang et al. [25] analyzed the effects of the equivalent damping coefficient, leakage coefficient, and proportional coefficient of the controller on the hydraulic screw-down system of the rolling mill. Results suggest that in the closed-loop state, when Proportional-Integral-Derivative (PID) controller parameters are fixed, the system will have parameter uncertainty due to the change of the equivalent damping coefficient and internal leakage coefficient.

Yuan et al. [26] investigated the dynamics, flow responses and power consumption theoretically and experimentally in hydraulic systems using the switched inertance hydraulic converter (SIHC). Results highlight the superiority of the SIHC in operation involving high pressures and delivery-flow rates. 
Qian et al. [27] proposed a static deformation-compensation method based on inclination sensor feedback for large-scale manipulators to reduce the deviation of the endpoint in manipulators with hydraulic actuation. Compared to the finite element method, the proposed method considers less boundary conditions, which are uncertain for flexible manipulators in most situations.

Zhu et al. [28] revealed the bifurcation characteristic of the load vertical vibration of the hydraulic automatic gauge control (HAGC) system through the investigation of the nonlinear factors such as excitation force, elastic force and damping force. Results point out that the resonance region can be effectively avoided by adjusting the nonlinear stiffness coefficient and the stability of the system will be promoted as well. In addition, Zhu et al. [29] described the function of the key position closed-loop system in HAGC. Results indicate that the absolute stability conditions of the position closed-loop system are derived whether the spool displacement is positive or negative.

Li et al. [30] introduced a new method for the evaluation of the blood cell damage and the observation of the real-time characteristics of blood flow patterns in vitro using rheometer and bionic microfluidic devices. The damaged erythrocytes were collected and injected into a bionic microfluidic device. Analysis of the captured images indicate that with the increase of shear stress suffered by the erythrocyte, the migration rate of damaged erythrocyte in bionic microchannel is significantly decreased.

Yuan et al. [31,32] investigated the natural frequency sensitivity and dynamic behaviors of the fire-fighting jet system. An adaptive gun-head design was proposed to achieve the fluid-structure interaction and discrete-continuous coupling characteristics and the sensitivity calculation formulas of the natural frequency was derived of the jet system to typical design parameters [31]. Focusing on the adaptive fire-fighting monitor, influence of the nonlinear fluid spring force on the dynamic characteristics was investigated. Results indicated that in the design of a fire-fighting system, the interval of the input shaft speed of the pump, and the pulsation frequency of the output fluid should be avoided [32].

Finally, in order to analyze the appropriate numerical simulation method for the investigation of the hydraulic performance, the mixing process and the flow law in the venturi injectors were considered by Li et al. [33]. Flow characteristics of the internal flow field obtained with and without the cavitation model were both compared with the experiments. Results indicate that the cavitation model has better agreement with experiments.

\section{Conclusions}

In this special issue, 33 papers are presented and they relate to smart flow control in pumps, valves, microfluidics and mechatronic systems. We believe that smart flow control, especially at microscales, will become more important and useful in the near future.

We would like to express our heartfelt gratitude to all the scientific contributors of the papers submitted to this Special Issue.

Author Contributions: Conceptualization, J.-y.Q. and J.Z.; Methodology, J.-y.Q. and Z.W.; Data Curation, J.-y.Q., J.Z. and Z.W.; Writing—Original Draft Preparation, J.-y.Q.; Writing—Review \& Editing, J.-y.Q. and B.S. All authors have read and agreed to the published version of the manuscript.

Funding: This research was funded by the National Natural Science Foundation of China, through grant number 51922093 and 51805470; the National Key R\&D Program of China, through grant number 2019YFB2005101; and the Yucai Project of Zhejiang Association for Science and Technology.

Conflicts of Interest: The authors declare no conflict of interest.

\section{References}

1. Bai, L.; Zhou, L.; Han, C.; Zhu, Y.; Shi, W. Numerical Study of Pressure Fluctuation and Unsteady Flow in a Centrifugal Pump. Processes 2019, 7, 354. [CrossRef]

2. Cao, W.; Jia, Z.; Zhang, Q. Near-Wall Flow Characteristics of a Centrifugal Impeller with Low Specific Speed. Processes 2019, 7, 514. [CrossRef] 
3. Si, Q.; Wang, B.; Yuan, J.; Huang, K.; Lin, G.; Wang, C. Numerical and Experimental Investigation on Radiated Noise Characteristics of the Multistage Centrifugal Pump. Processes 2019, 7, 793. [CrossRef]

4. Si, Q.; Shen, C.; Ali, A.; Cao, R.; Yuan, J.; Wang, C. Experimental and Numerical Study on Gas-Liquid Two-Phase Flow Behavior and Flow Induced Noise Characteristics of Radial Blade Pumps. Processes 2019, 7, 920. [CrossRef]

5. Wang, C.; Hu, B.; Zhu, Y.; Wang, X.; Luo, C.; Cheng, L. Numerical Study on the Gas-Water Two-Phase Flow in the Self-Priming Process of Self-Priming Centrifugal Pump. Processes 2019, 7, 330. [CrossRef]

6. Jiao, W.; Cheng, L.; Xu, J.; Wang, C. Numerical Analysis of Two-Phase Flow in the Cavitation Process of a Waterjet Propulsion Pump System. Processes 2019, 7, 690. [CrossRef]

7. Luo, C.; Liu, H.; Cheng, L.; Wang, C.; Jiao, W.; Zhang, D. Unsteady Flow Process in Mixed Waterjet Propulsion Pumps with Nozzle Based on Computational Fluid Dynamics. Processes 2019, 7, 910. [CrossRef]

8. Cao, W.; Jia, Z.; Zhang, Q. The Simulation of Vortex Structures Induced by Different Local Vibrations at the Wall in a Flat-Plate Laminar Boundary Layer. Processes 2019, 7, 563. [CrossRef]

9. Jin, Y.; He, X.; Zhang, Y.; Zhou, S.; Chen, H.; Liu, C. Numerical and Experimental Investigation of External Characteristics and Pressure Fluctuation of a Submersible Tubular Pumping System. Processes 2019, 7, 949. [CrossRef]

10. Xue, L.; Wu, S.; Xu, Y.; Ma, D. A Simulation-Based Multi-Objective Optimization Design Method for Pump-Driven Electro-Hydrostatic Actuators. Processes 2019, 7, 274. [CrossRef]

11. Zhang, J.; Liu, B.; LÜ, R.; Yang, Q.; Dai, Q. Study on Oil Film Characteristics of Piston-Cylinder Pair of Ultra-High Pressure Axial Piston Pump. Processes 2020, 8, 68. [CrossRef]

12. Zheng, Z.; Li, X.; Zhu, Y. A Fault Feature Extraction Method for the Fluid Pressure Signal of Hydraulic Pumps Based on Autogram. Processes 2019, 7, 695. [CrossRef]

13. Zheng, Z.; Wang, Z.; Zhu, Y.; Tang, S.; Wang, B. Feature Extraction Method for Hydraulic Pump Fault Signal Based on Improved Empirical Wavelet Transform. Processes 2019, 7, 824. [CrossRef]

14. Lei, Y.; Jiang, W.; Jiang, A.; Zhu, Y.; Niu, H.; Zhang, S. Fault Diagnosis Method for Hydraulic Directional Valves Integrating PCA and XGBoost. Processes 2019, 7, 589. [CrossRef]

15. Liu, Y.; Lu, L.; Zhu, K. Numerical Analysis of the Diaphragm Valve Throttling Characteristics. Processes 2019, 7, 671. [CrossRef]

16. Lu, L.; Long, S.; Zhu, K. A Numerical Research on Vortex Street Flow Oscillation in the Double Flapper Nozzle Servo Valve. Processes 2019, 7, 721. [CrossRef]

17. Lu, L.; Xue, Q.; Zhang, M.; Liu, L.; Wu, Z. Non-Structural Damage Verification of the High Pressure Pump Assembly Ball Valve in the Gasoline Direct Injection Vehicle System. Processes 2019, 7, 857. [CrossRef]

18. Qiu, C.; Jiang, C.-H.; Zhang, H.; Wu, J.-Y.; Jin, Z.-J. Pressure Drop and Cavitation Analysis on Sleeve Regulating Valve. Processes 2019, 7, 829. [CrossRef]

19. Wu, H.; Li, J.-Y.; Gao, Z.-X. Flow Characteristics and Stress Analysis of a Parallel Gate Valve. Processes 2019, 7, 803. [CrossRef]

20. Xu, X.-G.; Liu, T.-Y.; Li, C.; Zhu, L.; Li, S.-X. A Numerical Analysis of Pressure Pulsation Characteristics Induced by Unsteady Blood Flow in a Bileaflet Mechanical Heart Valve. Processes 2019, 7, 232. [CrossRef]

21. Qian, J.-Y.; Chen, M.-R.; Wu, Z.; Jin, Z.-J.; Sunden, B. Effects of a Dynamic Injection Flow Rate on Slug Generation in a Cross-Junction Square Microchannel. Processes 2019, 7, 765. [CrossRef]

22. Zhang, J.; Yu, X.; Tu, S.-T. Lattice Boltzmann Simulation on Droplet Flow through 3D Metal Foam. Processes 2019, 7, 877. [CrossRef]

23. Li, C.; Wu, W.; Liu, Y.; Hu, C.; Zhou, J. Analysis of Air-Oil Flow and Heat Transfer inside a Grooved Rotating-Disk System. Processes 2019, 7, 632. [CrossRef]

24. Guan, C.-B.; Shen, Y.; Yao, Z.-P.; Wang, Z.-L.; Zhang, M.-J.; Nan, K.; Hui, H.-H. Design, Simulation, and Experiment of an LTCC-Based Xenon Micro Flow Control Device for an Electric Propulsion System. Processes 2019, 7, 862. [CrossRef]

25. Zhang, Y.; Jiang, W.; Zhu, Y.; Li, Z. Research on the Vertical Vibration Characteristics of Hydraulic Screw Down System of Rolling Mill under Nonlinear Friction. Processes 2019, 7, 792. [CrossRef]

26. Yuan, C.; Mao Lung, V.L.; Plummer, A.; Pan, M. Theoretical and Experimental Studies of a Digital Flow Booster Operating at High Pressures and Flow Rates. Processes 2020, 8, 211. [CrossRef] 
27. Qian, J.; Su, Q.; Zhang, F.; Ma, Y.; Fang, Z.; Xu, B. Static Deformation-Compensation Method Based on Inclination-Sensor Feedback for Large-Scale Manipulators with Hydraulic Actuation. Processes 2020, 8, 81. [CrossRef]

28. Zhu, Y.; Tang, S.; Wang, C.; Jiang, W.; Yuan, X.; Lei, Y. Bifurcation Characteristic Research on the Load Vertical Vibration of a Hydraulic Automatic Gauge Control System. Processes 2019, 7, 718. [CrossRef]

29. Zhu, Y.; Tang, S.; Wang, C.; Jiang, W.; Zhao, J.; Li, G. Absolute Stability Condition Derivation for Position Closed-Loop System in Hydraulic Automatic Gauge Control. Processes 2019, 7, 766. [CrossRef]

30. Li, D.; Li, G.; Chen, Y.; Man, J.; Wu, Q.; Zhang, M.; Chen, H.; Zhang, Y. The Impact of Erythrocytes Injury on Blood Flow in Bionic Arteriole with Stenosis Segment. Processes 2019, 7, 372. [CrossRef]

31. Yuan, X.; Zhu, X.; Wang, C.; Zhang, L.; Zhu, Y. Natural Frequency Sensitivity Analysis of Fire-Fighting Jet System with Adaptive Gun Head. Processes 2019, 7, 808. [CrossRef]

32. Yuan, X.; Zhu, X.; Wang, C.; Zhang, L.; Zhu, Y. Research on the Dynamic Behaviors of the Jet System of Adaptive Fire-Fighting Monitors. Processes 2019, 7, 952. [CrossRef]

33. Li, H.; Li, H.; Huang, X.; Han, Q.; Yuan, Y.; Qi, B. Numerical and Experimental Study on the Internal Flow of the Venturi Injector. Processes 2020, 8, 64. [CrossRef]

(C) 2020 by the authors. Licensee MDPI, Basel, Switzerland. This article is an open access article distributed under the terms and conditions of the Creative Commons Attribution (CC BY) license (http://creativecommons.org/licenses/by/4.0/). 